\title{
Internalized Homophobia, Depressive Symptoms, and Suicidal Ideation Among Lesbian, Gay, and Bisexual Adults in South Korea: An Age-Stratified Analysis
}

\author{
Hyemin Lee, MPH, ${ }^{1,2}$ Don Operario, MS, PhD, ${ }^{2}$ Horim Yi, MSW, ${ }^{1}$ Sungsub Choo, EdM, \\ and Seung-Sup Kim, MD, MPH, ScD $D^{1,3}$
}

\begin{abstract}
Purpose: Little research on internalized homophobia (IHP) and mental health among lesbian, gay, and bisexual (LGB) individuals has been done in South Korea, a context in which LGB populations widely experience societal stigma. This study examined the association between IHP and depressive symptoms and suicidal ideation among Korean LGB adults and investigated whether the association differed by participants' age.

Methods: Cross-sectional data from the "Rainbow Connection Project I - Korean Lesbian, Gay, \& Bisexual Adults' Health Study" were used for this study $(N=2178)$. Descriptive analyses were conducted to explore the distribution of IHP, depressive symptoms, and suicidal ideation across all covariates. Multivariate analyses were also used to investigate the overall and age-stratified associations between IHP and depressive symptoms and suicidal ideation.

Results: The prevalence of high IHP was greater among older LGB adults, whereas the prevalence of depressive symptoms and suicidal ideation was higher among younger LGB adults. LGB individuals with high IHP levels had significantly greater risk of depressive symptoms than those with low IHP levels. In the age-stratified analyses, the associations between IHP and depressive symptoms were statistically significant only among older LGB adults with high IHP levels. Regarding suicidal ideation, the overall association was statistically significant in the full sample, but not in the age-stratified analyses.

Conclusion: These findings suggest that mental health interventions are needed for LGB adults who have high IHP levels, especially among older generations. Furthermore, greater efforts are needed to enact protective legislation for sexual minority individuals in South Korea.
\end{abstract}

Keywords: depressive symptoms, internalized homophobia, LGB, sexual minority, South Korea, suicidal ideation

\section{Introduction}

$\mathbf{M}$ ANY HEALTH DISPARITIES between sexual minority and heterosexual individuals can be attributed to negative social experiences that lesbian, gay, and bisexual (LGB) people have endured due to their sexual orientation, a phenomenon referred to as minority stress. ${ }^{1,2}$ Internalized homophobia (IHP), a common source of minority stress, is defined as the process whereby LGB people direct societal homophobic attitudes toward themselves. ${ }^{2,3}$ The literature has indicated that IHP is related to mental health problems, including depressive symptoms and suicidal behaviors. ${ }^{4-8}$
A meta-analysis of 31 empirical research studies investigating the association between IHP and mental health suggested that age could be an effect modifier in the association between IHP and negative mental health outcomes. ${ }^{7}$ The aforementioned study revealed that the association of IHP with poor mental health became stronger as the participants' age increased. ${ }^{7}$ Two explanations for this association were offered. First, older LGB individuals may have experienced more accumulated exposure to stigma and greater opportunities for IHP to affect mental health over time than those who were younger. Second, older LGB individuals may have been affected by living through more

\footnotetext{
${ }^{1}$ Department of Public Health Sciences, Graduate School of Korea University, Seoul, Republic of Korea.

${ }^{2}$ Department of Behavioral and Social Sciences, Brown University School of Public Health, Providence, Rhode Island.

${ }^{3}$ Department of Social and Behavioral Sciences, Harvard T.H. Chan School of Public Health, Boston, Massachusetts.
} 
hostile and less inclusive time periods than younger LGB individuals who, by comparison, have benefited from greater social inclusivity. However, this already mentioned study also pointed out that the findings should be interpreted cautiously due to the age heterogeneity within the available studies for the meta-analysis. ${ }^{7}$ Therefore, the study highlighted the need for future research to clearly identify the role of age in the association between IHP and mental health among LGB individuals.

In South Korea (hereafter Korea), societal attitudes toward sexual minority individuals have been changing incrementally in a positive direction during the past 20 years from 1994 to $2014 .{ }^{9}$ However, relative to many Western contexts, explicitly negative and sometimes hostile attitudes toward LGB populations persist in Korea. In a 2013 global study of national attitudes toward homosexuality, Korea was the least supportive among all high-income countries included in the report. ${ }^{10}$ Some reasons for overt homophobia and negativity include the Confucianist belief systems that have historically shaped Korean culture and the influence of conservative Christian groups throughout society and in key institutions, such as education, which both consider homosexuality as abnormal and immoral. ${ }^{9,11}$ Recently, an antiLGBT movement, led by conservative Christian groups, has been growing quickly in Korea. ${ }^{11,12}$ In addition, marriage equality has not been achieved and antidiscrimination laws, which address discrimination on the basis of sexual orientation, have not been institutionalized in Korea as of 2019. ${ }^{13}$ In such sociopolitical environments, LGB people might experience more minority stress and have fewer coping resources, which contribute to poor mental and physical health. $^{2}$

A recent study showed critical health disparities among Korean LGB individuals. ${ }^{14}$ In a large population survey comparing health indicators between 2335 Korean LGB adults and the general population, the age-standardized prevalence of depressive symptoms among LGB participants was five to seven times higher than that of the general population. ${ }^{14}$ Regarding suicidal behaviors, the age-standardized prevalence was much higher, 6 to 37 times, for LGB individuals than for the general population. ${ }^{14}$ In light of data showing that Korea has the highest suicide rate among the Organisation for Economic Co-operation and Development (OECD) countries, ${ }^{15}$ urgent attention should focus on the mental health indicators, especially suicide, of Korean sexual minority populations.

Furthermore, only a few studies have examined IHP and its impact on the mental health of LGB populations in Korea. ${ }^{16,17}$ A study of 237 Korean gay men and lesbian women found that IHP was not associated with mental health outcomes, but was positively associated with physical health. ${ }^{16}$ Another study of 2205 Korean adolescents found that $8.0 \%(N=177)$ of participants reported same-sex attraction among whom the association between homophobia and depression was not statistically significant after adjusting for potential confounders. ${ }^{17}$ There were methodological limitations to these studies. First, the studies did not use an IHP scale designed for sexual minority individuals, but instead used a homophobia scale originally designed for heterosexual individuals. Second, due to their small sample sizes, these studies may lack statistical power to investigate the association between IHP and mental health.
To fill the knowledge gaps in previous studies, this study aimed to investigate the association between IHP and depressive symptoms and suicidal ideation using the IHP scale, which was developed for sexual minority individuals and has been used extensively in related literature, ${ }^{3,7}$ with a large sample of Korean LGB adults. In addition, this study aimed to examine whether the association differs by participants' age.

\section{Methods}

\section{Data and participants}

The data for this study were from the "Rainbow Connection Project I - Korean Lesbian, Gay, \& Bisexual Adults' Health Study," and the sample of LGB adults was collected using nonprobability sampling methods. Despite being the largest survey on the health of the LGB population in Korea to date, the survey is not a nationally representative sample of Korean LGB individuals. The data, collected in 2016, were from a cross-sectional online survey designed to assess indicators of health status as well as sociodemographic characteristics of LGB adults in Korea. Participants were Koreans, 19 years or older, and self-identified as LGB. This study obtained informed consent from LGB adults who participated in the survey and was approved by the institutional review board of Korea University (1040548-KU-IRB-16-191-A-1). Among 2430 individuals who completed the survey, a total of 2178 LGB participants were included in the final analysis after excluding those who did not provide informed consent for participation or academic use of the data $(N=17)$ and those who had missing values on IHP, depressive symptoms, suicidal ideation $(N=88)$, and sociodemographic variables including age and gender $(N=147)$. Additional details regarding the study are provided elsewhere. ${ }^{14}$

\section{Measures}

The Internalized Homophobia Scale is composed of nine items that assess internalized negative views that LGB individuals have of themselves, ${ }^{3,7}$ originally developed for gay men. ${ }^{3}$ The scale was translated into Korean and included in the study. To ensure parity between the original measure and the Korean-translated version, a cross-cultural adaptation method was used. ${ }^{18,19}$ According to the guidelines for the cross-cultural adaptation, five steps were taken: (1) translation by two independent translators, (2) synthesis, (3) backtranslation by two other bilingual individuals, (4) expert's review, and (5) finalization. ${ }^{18}$

In the survey, LGB adults were asked how often they have negative feelings regarding their sexual identity using the Korean-translated version of the IHP scale (Supplementary Table S1). In Table 1, the items of the scale are presented in English. Respondents rated the frequency of having those feelings in the past year, with the following response options: "never (1)," "rarely (2)," "sometimes (3)," or "often (4)." For the analysis, IHP was categorized into two groups based on the overall mean score. Prior studies showed an acceptable internal consistency reliability coefficient of IHP (Cronbach's $\alpha=0.79) .{ }^{1}$ In this study, the Korean version of the scale exhibited a high internal consistency (Cronbach's $\alpha=0.85$ ). 
Table 1. Experiences of Internalized Homophobia (IHP) Assessed by the IHP Scale Among 2178 Lesbian, Gay, and Bisexual Adults in Korea

\begin{tabular}{lcc}
\hline Variable & Mean (SD) & $\begin{array}{c}\text { Responses of "sometimes" } \\
\text { and "often" } \mathrm{n}(\%)\end{array}$ \\
\hline $\begin{array}{l}\text { I wish I weren't lesbian/gay/bisexual. } \\
\text { If someone offered me the chance to be completely heterosexual, I would }\end{array}$ & $1.65(0.90)$ & $374(17.2)$ \\
$\quad \begin{array}{l}\text { accept the chance. } \\
\text { I feel that being lesbian/gay/bisexual is a personal shortcoming for me. }\end{array}$ & $1.55(0.84)$ & $397(18.2)$ \\
$\begin{array}{l}\text { I often feel it best to avoid personal or social involvement with other } \\
\text { lesbian/gay/bisexual individuals. }\end{array}$ & $1.51(0.82)$ & $288(14.2)$ \\
$\begin{array}{l}\text { I have tried to become more sexually attracted to people who are the } \\
\quad \text { opposite sex. }\end{array}$ & $1.50(0.77)$ & $269(12.4)$ \\
$\begin{array}{l}\text { I have tried to stop being attracted to people who are the same sex as me in } \\
\text { general. }\end{array}$ & $1.47(0.79)$ & $279(12.8)$ \\
$\begin{array}{l}\text { I wish that I could develop more erotic feelings about people who are the } \\
\quad \text { opposite sex. }\end{array}$ & $1.39(0.73)$ & $203(9.3)$ \\
$\begin{array}{l}\text { I feel alienated from myself because of being lesbian/gay/bisexual. } \\
\text { I would like to get professional help to change my sexual orientation from } \\
\text { lesbian/gay/bisexual to straight. }\end{array}$ & $1.29(0.67)$ & $165(7.6)$ \\
\hline
\end{tabular}

The Korean-translated version of the IHP scale is available as Supplementary Table S1.

IHP, internalized homophobia; SD, standard deviation.

Depressive symptoms were assessed using the Center for Epidemiologic Studies Depression Scale-20, ${ }^{20}$ which has been previously validated for use with the Korean population. ${ }^{21}$ For each type of depressive symptom listed, respondents indicated how often they experienced them within the last week: $<1$ day (0), 1 or 2 days (1), 3 or 4 days (2), and $\geq 5$ days (3). Values for the summed score ranged from 0 to 60 , and a score $\geq 16$ points was categorized as having depressive symptoms. Suicidal ideation was measured by a single question, "have you ever had a serious thought about dying by suicide over the past 12 months?" Respondents could answer either "yes" or "no."

Sociodemographic characteristics included age, sexual identity, residential area, educational and income level, and employment status. Age was divided into three groups: 19$29,30-39$, and 40-69 years old. Gender was categorized as either woman or man. Sexual orientation was assessed using a single question, "How do you identify your sexual orientation?" Participants reported their sexual orientation as lesbian/gay or bisexual. Sexual identity was defined using respondents' gender and sexual orientation to create four categories: lesbian women, gay men, bisexual women, and bisexual men. Residential area was categorized into two groups: metropolitan area or rural area. Educational level was divided into four categories: high school graduate or less, 2-year college student/dropout/graduate, 4-year college student/dropout/graduate, and graduate school student/dropout/graduate or more. Annual household income (unit $=1000 \mathrm{KRW} ; 1000 \mathrm{KRW}=$ approximately $0.88 \mathrm{USD}$ ) was categorized into five groups: $<10,000,10,000-19,999$, 20,000-29,999, 30,000-49,999, and $\geq 50,000$. Employment status was identified as unemployed, nonpermanent employment, permanent employment, self-employed worker, or unpaid family worker.

\section{Statistical analyses}

Descriptive analyses were conducted to quantify the prevalence of IHP, depressive symptoms within the last week, and suicidal ideation over the past 12 months across all covariates. Multivariate analyses were used to investigate the overall and age-stratified associations between IHP and depressive symptoms and suicidal ideation. All covariates were included in the adjusted model. As the prevalence of depressive symptoms and suicidal ideation was much more than $20 \%$ in our study, we used a modified Poisson regression model with a log-link and robust sandwich variance estimator. ${ }^{22}$ All statistical analyses were done using STATA/SE version 15.0 (StataCorp LLC, College Station, TX) and prevalence ratios (PRs) were estimated with 95\% confidence intervals (CIs).

\section{Results}

Table 1 provides the mean scores and standard deviations for each IHP item, as well as the number and percentage of participants who responded to each item with "sometimes" and "often." The item with the highest mean score was "I wish I weren't lesbian/gay/bisexual" (mean: 1.65). The percentage of those who responded to the item, "If someone offered me the chance to be completely heterosexual, I would accept the chance," with "sometimes" and "often" was the highest $(18.2 \%)$.

The distribution of the study population and the estimated prevalence of IHP by sociodemographic characteristics are presented in Table 2. The prevalence of high IHP was higher among men, especially bisexual men, than it was among women. Prevalence of high IHP was also higher among participants aged 40-69 years than it was among those aged 1929 and 30-39 years. Prevalence estimates for depressive symptoms and suicidal ideation by sociodemographic characteristics are also presented in Table 2 . The overall prevalence rate was $45.0 \%$ for depressive symptoms and $34.8 \%$ for suicidal ideation. The prevalence of depressive symptoms and suicidal ideation was higher among women, younger LGB adults, and those with lower educational and income level, than their counterparts.

Table 3 shows the associations between IHP and depressive symptoms among Korean LGB adults. Compared with 
Table 2. Prevalence and Distribution of Internalized Homophobia, Depressive Symptoms, and Suicidal Ideation by Key Covariates Among 2178 Lesbian, Gay, and Bisexual Adults in Korea

\begin{tabular}{|c|c|c|c|c|c|c|c|}
\hline & Overall & High I & $H P$ & Depressive & ymptoms & Suicidal ic & deation \\
\hline & $\mathrm{n}(\%)$ & $\mathrm{n}(\%)$ & $\mathrm{p}$ & $\mathrm{n}(\%)$ & $\mathrm{p}$ & $\mathrm{n}(\%)$ & $\mathrm{p}$ \\
\hline Gender & & & $<0.001$ & & $<0.001$ & & $<0.001$ \\
\hline Man & $965(44.3)$ & $571(59.2)$ & & $330(34.2)$ & & $253(26.2)$ & \\
\hline Woman & $1213(55.7)$ & $538(44.4)$ & & $650(53.6)$ & & 504 (41.6) & \\
\hline Sexual identity & & & $<0.001$ & & $<0.001$ & & $<0.001$ \\
\hline Gay man & $851(39.1)$ & $487(57.2)$ & & $285(33.5)$ & & $221(26.0)$ & \\
\hline Bisexual man & $114(5.2)$ & $84(73.7)$ & & $45(39.5)$ & & $32(28.1)$ & \\
\hline Lesbian woman & $582(26.7)$ & $260(44.7)$ & & $275(47.3)$ & & $201(34.5)$ & \\
\hline Bisexual woman & $631(29.0)$ & $278(44.1)$ & & $375(59.4)$ & & $303(48.0)$ & \\
\hline Age (years) & & & 0.046 & & $<0.001$ & & $<0.001$ \\
\hline $19-29$ & 1549 (71.1) & $766(49.5)$ & & $771(49.8)$ & & $585(37.8)$ & \\
\hline $30-39$ & 426 (19.6) & $225(52.8)$ & & $149(35.0)$ & & $121(28.4)$ & \\
\hline $40-69$ & $203(9.3)$ & $118(58.1)$ & & $60(29.6)$ & & $51(25.1)$ & \\
\hline Residential area & & & 0.621 & & 0.205 & & 0.067 \\
\hline Metro & $1462(67.1)$ & 739 (50.6) & & $644(44.1)$ & & $489(33.5)$ & \\
\hline Rural area & 716 (32.9) & $370(51.7)$ & & $336(46.9)$ & & $268(37.4)$ & \\
\hline Education level & & & 0.780 & & $<0.001$ & & $<0.001$ \\
\hline graduate or less & $181(8.3)$ & $93(51.4)$ & & $100(55.3)$ & & $95(52.5)$ & \\
\hline 2-Year college stud & $260(11.9)$ & $126(48.5)$ & & $143(55.0)$ & & $106(40.8)$ & \\
\hline 4-Year collę & $1490(68.4)$ & $759(50.9)$ & & $646(43.4)$ & & $492(33.0)$ & \\
\hline $\begin{array}{l}\text { Graduate school student/dropout/ } \\
\text { graduate or more }\end{array}$ & $247(11.3)$ & $131(53.0)$ & & $91(36.8)$ & & $64(25.9)$ & \\
\hline Annual household income $\left(10^{3} \mathrm{KRW}\right)$ & & & 0.004 & & $<0.001$ & & 0.001 \\
\hline$<10,000$ & $363(16.7)$ & $198(54.6)$ & & $204(56.2)$ & & $153(42.2)$ & \\
\hline $10,000-19,999$ & $351(16.1)$ & $154(43.9)$ & & $196(55.8)$ & & $132(37.6)$ & \\
\hline $20,000-29,999$ & $410(18.8)$ & $191(46.6)$ & & $191(46.6)$ & & $144(35.1)$ & \\
\hline $30,000-49,999$ & $572(26.3)$ & $307(53.7)$ & & $234(40.9)$ & & $189(33.0)$ & \\
\hline$\geq 50,000$ & $482(22.1)$ & $259(53.7)$ & & $155(32.2)$ & & $139(28.8)$ & \\
\hline Employment status & & & 0.121 & & $<0.001$ & & $<0.001$ \\
\hline Unemployed & $948(43.5)$ & $473(49.9)$ & & $502(53.0)$ & & $375(39.6)$ & \\
\hline Nonpe & $564(25.9)$ & $294(52.1)$ & & $181(32.1)$ & & $145(25.7)$ & \\
\hline Perma & $541(24.8)$ & $285(52.7)$ & & $259(47.9)$ & & $195(36.0)$ & \\
\hline Self-employed/employer & $104(4.8)$ & $43(41.4)$ & & $23(22.1)$ & & $32(30.8)$ & \\
\hline Unpaid family worker & $21(1.0)$ & $14(66.7)$ & & $15(71.4)$ & & $10(47.6)$ & \\
\hline
\end{tabular}

All $p$ values correspond to the chi-square test of association.

KRW, Korean Won.

Table 3. The Overall and Age-Stratified Associations Between Internalized Homophobia and Depressive Symptoms Among 2178 Lesbian, Gay, and Bisexual Adults in Korea

\begin{tabular}{|c|c|c|c|c|c|c|}
\hline \multirow[b]{2}{*}{ Internalized homophobia } & \multirow{2}{*}{$\begin{array}{c}\text { Overall } \\
\mathrm{n}(\%)\end{array}$} & \multirow{2}{*}{$\frac{\text { Prevalence }}{\mathrm{n}(\%)}$} & \multicolumn{2}{|c|}{ Unadjusted } & \multicolumn{2}{|c|}{ Adjusted $^{\mathrm{a}}$} \\
\hline & & & $P R$ & $95 \% C I$ & $P R$ & $95 \% C I$ \\
\hline Low & $1069(49.1)$ & $453(42.4)$ & 1 & Reference & 1 & Reference \\
\hline High & $1109(50.9)$ & $527(47.5)$ & $1.12^{*}$ & $1.02-1.23$ & $1.20 * * *$ & $1.10-1.31$ \\
\hline \multicolumn{7}{|c|}{ LGB adults aged $19-29$ years $(N=1549)$} \\
\hline Low & $783(50.6)$ & $382(48.8)$ & 1 & Reference & 1 & Reference \\
\hline High & $766(49.5)$ & $389(50.8)$ & 1.04 & $0.94-1.15$ & 1.10 & $1.00-1.21$ \\
\hline \multicolumn{7}{|c|}{ LGB adults aged $30-39$ years $(N=426)$} \\
\hline Low & $201(47.2)$ & $57(28.4)$ & 1 & Reference & 1 & Reference \\
\hline High & $225(52.8)$ & $92(40.9)$ & $1.44 * *$ & $1.10-1.89$ & $1.43 * *$ & $1.10-1.86$ \\
\hline \multicolumn{7}{|c|}{ LGB adults aged 40-69 years $(N=203)$} \\
\hline Low & $85(41.9)$ & $14(16.5)$ & 1 & Reference & 1 & Reference \\
\hline High & $118(58.1)$ & $46(39.0)$ & $2.37 * * *$ & $1.39-4.02$ & $2.29 * * *$ & $1.39-3.75$ \\
\hline
\end{tabular}

$* p<0.05, * * p<0.01, * * * p<0.001$.

aAjusted for sexual identity, residential area, educational level, annual household income, and employment status.

CI, confidence interval; LGB, lesbian, gay, and bisexual; PR, prevalence ratio. 
Table 4. The Overall and Age-Stratified Associations Between Internalized Homophobia and Suicidal Ideation Among 2178 Lesbian, Gay, and Bisexual Adults in Korea

\begin{tabular}{|c|c|c|c|c|c|c|}
\hline \multirow[b]{2}{*}{ Internalized homophobia } & \multirow{2}{*}{$\begin{array}{c}\text { Overall } \\
\mathrm{n}(\%)\end{array}$} & \multirow{2}{*}{$\frac{\text { Prevalence }}{\mathrm{n}(\%)}$} & \multicolumn{2}{|c|}{ Unadjusted } & \multicolumn{2}{|c|}{ Adjusted $^{\mathrm{a}}$} \\
\hline & & & $P R$ & $95 \% C I$ & $P R$ & $95 \% C I$ \\
\hline Low & $1069(49.1)$ & $361(33.8)$ & 1 & Reference & 1 & Reference \\
\hline High & 1109 (50.9) & $396(35.7)$ & 1.06 & $0.94-1.19$ & $1.13^{*}$ & $1.01-1.27$ \\
\hline \multicolumn{7}{|c|}{ LGB adults aged $19-29$ years $(N=1549)$} \\
\hline Low & $783(50.6)$ & $295(37.7)$ & 1 & Reference & 1 & Reference \\
\hline High & $766(49.5)$ & $290(37.9)$ & 1.00 & $0.88-1.14$ & 1.07 & $0.94-1.21$ \\
\hline \multicolumn{7}{|c|}{ LGB adults aged $30-39$ years $(N=426)$} \\
\hline Low & $201(47.2)$ & $48(23.9)$ & 1 & Reference & & Reference \\
\hline High & $225(52.8)$ & $73(32.4)$ & 1.36 & $1.00-1.85$ & 1.36 & $1.00-1.86$ \\
\hline \multicolumn{7}{|c|}{ LGB adults aged $40-69$ years $(N=203)$} \\
\hline Low & 85 (41.9) & $18(21.2)$ & 1 & Reference & 1 & Reference \\
\hline High & $118(58.1)$ & $33(28.0)$ & 1.32 & $0.80-2.18$ & 1.20 & $0.71-2.02$ \\
\hline
\end{tabular}

$* p<0.05$.

aAdjusted for sexual identity, residential area, educational level, annual household income, and employment status.

LGB individuals with low IHP levels, prevalence of depressive symptoms was statistically significantly higher among those with high IHP levels (adjusted prevalence ratio [aPR]: $1.20,95 \% \mathrm{CI}: 1.10-1.31)$ after adjusting for potential confounders. When stratified by age, the associations between IHP and depressive symptoms were statistically significant for those aged 30-39 years (aPR: 1.43, 95\% CI: 1.10-1.86) and 40-69 years (aPR: 2.29, 95\% CI: 1.39 3.75) among LGB adults with high IHP levels. However, the association was not significant for LGB adults aged 19-29 years among those with high IHP.

Table 4 presents the associations between IHP and suicidal ideation. Prevalence of suicidal ideation was statistically significantly higher among LGB adults with high IHP levels than those with low IHP levels (aPR: 1.13, 95\% CI: 1.011.27). In the age-stratified analyses, we found no significant association between IHP and suicidal ideation among LGB individuals with high IHP levels across all age groups. Among LGB adults with high IHP, the PRs of suicidal ideation were 1.07 (95\% CI: 0.94-1.21), 1.36 (95\% CI: 1.00 1.86), and 1.20 (95\% CI: 0.71-2.02) for those aged 19-29, $30-39$, and 40-69 years, respectively.

\section{Discussion}

Our findings indicate that older LGB adults experienced higher prevalence of high IHP relative to younger LGB adults. By contrast, younger LGB participants experienced higher prevalence of both depressive symptoms and suicidal ideation than older participants. The latter is consistent with findings well established in the literature, which show that older adults are less likely to report adverse mental health outcomes than younger adults. ${ }^{23,24}$ With regard to younger LGB adults, several possible factors could have contributed to their vulnerability for adverse mental health outcomes relative to older LGB adults. Younger LGB individuals are generally more concerned about disclosing their sexual identity to their family and friends, which can be a considerable stressor to them, than older LGB individuals who have had a longer life course to disclose their sexual identity. ${ }^{25}$ During the early coming-out process, younger LGB adults may face neg- ative reactions from others including rejection and victimization. ${ }^{26,27}$ Furthermore, they may have had fewer opportunities to develop effective coping strategies that could help them deal with mental health issues than older LGB adults. ${ }^{28,29}$

Consistent with findings reported in studies conducted elsewhere, ${ }^{4-8}$ there was a statistically significant association between IHP and depressive symptoms among Korean LGB participants. In the age-stratified analyses, IHP was associated with depressive symptoms in older LGB people, particularly LGB adults aged 40-69 years. However, IHP was not statistically significantly associated with depressive symptoms in younger LGB people, ages 19-29 years. Regarding suicidal ideation, we did not find any statistically significant associations in the age-stratified analyses. A meta-analytic review of 31 studies suggested that, when results were aggregated across studies, the estimated strength of association between IHP and mental health rose as participants' age increased. ${ }^{7}$ Our findings corroborate this trend among LGB adults in Korea. As noted, this association may reflect the greater number of years through which older Korean LGB adults have been exposed to IHP, having a resultant impact on their mental health. This effect may also be interpreted in light of historical changes in LGB inclusion and visibility, where older adults in Korea lived through periods that were less inclusive and in which LGB populations were less visible, relative to those experienced by their younger counterparts.

As exploratory analyses, we stratified the associations by participants' gender. In the gender-stratified analyses, significant associations between IHP and depressive symptoms were observed among gay/bisexual men and lesbian/bisexual women (Supplementary Table S2). Regarding suicidal ideation, the association was significant only among gay/bisexual men; no significant association was found among lesbian/bisexual women (Supplementary Table S3). These findings are partly consistent with the results of meta-analysis research demonstrating that gender does not moderate the relationship between IHP and mental health. ${ }^{7}$ Additional research, therefore, is needed to understand this pattern in the context of Korea's cultural norms regarding gender, sexuality, and mental health.

It is important to consider the findings from this research in the context of the current social climate of Korea, where 
instances of open hostility and aggression toward sexual minority individuals have been recently reported. In 2017, it was reported that the Korean army tracked down sexual minority soldiers and punished them, based on Article 92-6 of the Military Criminal Act, which is known for punishing homosexuality. ${ }^{13}$ The existence and practice of this law is problematic in Korea, where all men are required to serve 2 years of conscription. In 2018, a queer festival, also known as pride parade, in a large city in Korea was interrupted by anti-LGBT protesters. ${ }^{30}$ Those who supported the anti-LGBT movement blocked the parade route and attacked festival attendees. A study conducted a week later with the festival attendees $(N=305)$ found that $97.7 \%$ heard derogatory comments about sexual and gender minority individuals, $32.5 \%$ reported the experience of physical assault, such as pinching or hitting, and $51.8 \%$ reported the experience of sexual harassment or assault. ${ }^{31}$

Furthermore, it is difficult for sexual minority individuals in Korea to disclose their identity to important people in their lives and receive interpersonal support. Previous research found that only $21.8 \%$ and $10.8 \%$ of 3156 lesbian, gay, bisexual, transgender, and intersex (LGBTI) individuals came out to their mother and father, respectively. ${ }^{32}$ Given the hostile circumstances surrounding Korean LGB individuals, they might become vulnerable to internalizing negative attitudes and beliefs about sexual minority individuals, which makes it hard for them to affirm themselves.

\section{Limitations}

There are important limitations to this research. First, this study used a cross-sectional design that limits inferences about causality and temporality. Second, this study analyzed data from an online community-based survey using nonprobability sampling, which might further limit generalizability of the findings. Those who voluntarily participated in the online community-based survey were more likely to be out, visible, and accepting of their sexual orientation. ${ }^{2}$ Third, this study used a cross-cultural adaptation method to translate the IHP scale that was developed in the United States. There might be difficulty in reflecting nuanced local experiences of IHP among Korean LGB adults. Fourth, IHP, depressive symptoms, and suicidal ideation were self-reported in our study, which might be prone to self-report or recall bias. Lastly, this study did not use a two-step method to assess participants' gender, that is, asking questions about their assigned sex at birth in addition to their current gender identity. ${ }^{33}$ Consequently, some participants' gender might have been misclassified.

\section{Implications}

Despite the limitations of the study, this article contributes additional understanding about the prevalence of and associations between IHP and depressive symptoms and suicidal ideation among LGB adults in Korea. This study also has important policy implications for improving the health of Korean sexual minority individuals. Since the early 2000 s, the suicide rate in Korea has been the highest among the OECD member nations. ${ }^{15}$ A previous study demonstrated that suicidal ideation is $6-10$ times more prevalent among LGB adults than among the general population in Korea. ${ }^{14}$ Mental health interventions, therefore, should be implemented to lower IHP as well as improve mental health among LGB individuals in Korea.
Furthermore, due to the social climate described previously, there is a need to enact protective policies and advocate for inclusive practices to support Korean sexual minority individuals. Korea has been discussing antidiscrimination legislation since the mid-2000s; however, the legislative effort has been repeatedly undermined by anti-LGBT protesters and conservative Christian groups. ${ }^{34}$ Considering the crucial effect of protective policies and practices for sexual minority individuals, comprehensive antidiscrimination laws should be enacted urgently in Korea.

\section{Conclusion}

This study found that the association between IHP and depressive symptoms was statistically significant among LGB adults with high IHP levels. In the age-stratified analyses, only older LGB participants with high IHP levels had significantly greater risk of depressive symptoms compared with those with low IHP levels. A significant association between IHP and suicidal ideation was also found among the entire sample of LGB participants, although it was not observed in the age-stratified analyses. Our findings suggest that institutional interventions on mental health should be developed for LGB individuals who have high IHP levels, especially for those in older generations, and enhanced efforts are needed to enact protective legislation for Korean sexual minority individuals.

\section{Acknowledgments}

Many thanks to the members of the LGBTAIQP Suicide Prevention Project Connect-your-hearts of Chingusai for their assistance in survey development and promotion. The authors appreciate Dr. Ilan Meyer who reviewed and provided important feedback in the process of cross-cultural adaptation of the internalized homophobia scale as an expert.

\section{Author Disclosure Statement}

No competing financial interests exist.

\section{Funding Information}

This study was partially financially supported by the Korean Gay Men's Human Rights Group Chingusai and the Beautiful Foundation, the Ministry of Education of the Republic of Korea and National Research Foundation of Korea (NRF-2018S1A5B6075594), and Korea University Grant (K1711251).

\section{Supplementary Material}

Supplementary Table S1 Supplementary Table S2

Supplementary Table S3

\section{References}

1. Meyer IH: Minority stress and mental health in gay men. J Health Soc Behav 1995;36:38-56.

2. Meyer IH: Prejudice, social stress, and mental health in lesbian, gay, and bisexual populations: Conceptual issues and research evidence. Psychol Bull 2003;129:674-697.

3. Meyer IH, Dean L: Internalized homophobia, intimacy, and sexual behavior among gay and bisexual men. In: Psychological 
Perspectives on Lesbian and Gay Issues: Vol. 4. Stigma and Sexual Orientation: Understanding Prejudice Against Lesbians, Gay Men, and Bisexuals, 1st ed. Edited by Herek GM. Thousand Oaks, CA: Sage Publications, Inc., 1998, pp 160-186.

4. Davidson K, McLaren S, Jenkins M, et al.: Internalized homonegativity, sense of belonging, and depressive symptoms among Australian gay men. J Homosex 2017;64:450-465.

5. McLaren S: Gender, age, and place of residence as moderators of the internalized homophobia-depressive symptoms relation among Australian gay men and lesbians. J Homosex 2015;62:463-480.

6. McLaren S: The interrelations between internalized homophobia, depressive symptoms, and suicidal ideation among Australian gay men, lesbians, and bisexual women. J Homosex 2016;63:156-168.

7. Newcomb ME, Mustanski B: Internalized homophobia and internalizing mental health problems: A meta-analytic review. Clin Psychol Rev 2010;30:1019-1029.

8. Xu W, Zheng L, Xu Y, Zheng Y: Internalized homophobia, mental health, sexual behaviors, and outness of gay/bisexual men from Southwest China. Int J Equity Health 2017;16:36.

9. Youn G: Attitudinal changes toward homosexuality during the past two decades (1994-2014) in Korea. J Homosex 2018;65:100-116.

10. Kohut A, Wike R, Bell J, et al.: The global divide on homosexuality. Washington, DC: Pew Research Center, 2013. Available at https://www.pewresearch.org/wp-content/uploads/ sites/2/2014/05/Pew-Global-Attitudes-Homosexuality-ReportREVISED-MAY-27-2014.pdf Accessed August 23, 2019.

11. Lee NY, Baek JY: "Politics of disgust": Korean conservative protestant discourses against homosexuality. [In Korean] J Womens Study 2017;27:67-108.

12. Yi H, Kim S-S: Anti-LGBT movement in South Korea and the professionals on their side. [In Korean] Psychol Sex Rev 2016;7:83-85.

13. SOGILAW: Human Rights Situation of LGBTI in South Korea 2017, 1st ed. [In Korean]. Seoul: SOGILAW, 2018.

14. Yi H, Lee H, Park J, et al.: Health disparities between lesbian, gay, and bisexual adults and the general population in South Korea: Rainbow Connection Project I. Epidemiol Health 2017;39:e2017046.

15. Organisation for Economic Co-operation and Development. OECD Factbook 2015-2016: Economic, Environmental and Social Statistics. 2016. Available at https://www.oecd.org/ publications/oecd-factbook-18147364.htm Accessed August 23, 2019.

16. Shin S: A study on health related quality of life of gay men and lesbians in Korea. [In Korean] Health Soc Welfare Rev 2013;33:380-416.

17. Lee YS, Kwon BM, Lee DH: The relationships among homosexual experiences, sexual attitude, sexual homophobic, and depression in adolescents. [In Korean] J Rehabil Psychol 2012;19:129-147.

18. Beaton DE, Bombardier C, Guillemin F, Ferraz MB: Guidelines for the process of cross-cultural adaptation of selfreport measures. Spine (Phila Pa 1976) 2000;25:3186-3191.

19. Guillemin F, Bombardier C, Beaton D: Cross-cultural adaptation of health-related quality of life measures: Literature review and proposed guidelines. J Clin Epidemiol 1993; 46:1417-1432.

20. Radloff LS: The CES-D scale: A self-report depression scale for research in the general population. Appl Psychol Meas 1977;1:385-401.
21. Cho MJ, Kim KH: Diagnostic validity of the CES-D (Korean version) in the assessment of DSM-III-R major depression. J Korean Neuropsychiatry Assoc 1993;32:381-399.

22. Zou G: A modified poisson regression approach to prospective studies with binary data. Am J Epidemiol 2004;159:702-706.

23. Fiske A, Wetherell JL, Gatz M: Depression in older adults. Annu Rev Clin Psychol 2009;5:363-389.

24. Wolitzky-Taylor KB, Castriotta N, Lenze EJ, et al.: Anxiety disorders in older adults: A comprehensive review. Depress Anxiety 2010;27:190-211.

25. Pachankis JE, Cochran SD, Mays VM: The mental health of sexual minority adults in and out of the closet: A populationbased study. J Consult Clin Psychol 2015;83:890-901.

26. Baams L, Grossman AH, Russell ST: Minority stress and mechanisms of risk for depression and suicidal ideation among lesbian, gay, and bisexual youth. Dev Psychol 2015;51:688-696.

27. Ryan C, Huebner D, Diaz RM, Sanchez J: Family rejection as a predictor of negative health outcomes in White and Latino lesbian, gay, and bisexual young adults. Pediatrics 2009; 123:346-352.

28. DiFulvio GT: Sexual minority youth, social connection and resilience: From personal struggle to collective identity. Soc Sci Med 2011;72:1611-1617.

29. Fredriksen-Goldsen KI, Kim HJ, Shiu C, et al.: Successful aging among LGBT older adults: Physical and mental health-related quality of life by age group. Gerontologist 2015;55:154-168.

30. Lee C: [Newsmaker] Queer festival severely delayed by violent anti-gay protests in Korean port city. The Korea Herald, 2018. Available at www.koreaherald.com/view.php?ud= 20180909000245 Accessed August 23, 2019.

31. Choo S: Festival attendees' experiences of hate crime victimization and its public health implications. In: Assessment of Hate Crimes in the Korean Society and the Resolutions: Based on What Happened at the 2018 Incheon Queer Cultural Festival, 1st ed. [In Korean] Edited by Incheon Queer Cultural Festival Emergency Board and MinbyunLawyers for a Democratic Society Incheon Chapter. Seoul: The National Assembly of the Republic of Korea, 2019, pp 27-38.

32. SOGILAW. Korean LGBTI Community Social Needs Survey Final Report. [In Korean] Seoul, Korean Gay Men's Human Rights Group Chingusai, 2014.

33. Tate CC, Ledbetter JN, Youssef CP: A two-question method for assessing gender categories in the social and medical sciences. J Sex Res 2013;50:767-776.

34. Lee JH: Disputes and perspectives on sex orientation in the legislation of antidiscrimination law in Korea. [In Korean] Chung-Ang Law Rev 2014;16:107-139.

Address correspondence to: Seung-Sup Kim, MD, MPH, ScD Department of Public Health Sciences Graduate School of Korea University 368 Hana Science Hall B Anam-ro 145 Seongbuk-gu, Seoul 02841 Republic of Korea

E-mail: ssk3@korea.ac.kr 